\title{
SECTIONAL CURVATURE IN PIECEWISE LINEAR MANIFOLDS
}

\author{
BY DAVID A. STONE ${ }^{1}$ \\ Communicated by William Browder, February 13, 1973
}

A metric complex $M$ is a connected, locally-finite simplicial complex linearly embedded in some Euclidean space $R^{l}$. Metric complexes $M$ and $M^{\prime}$ are isometric if they have subdivisions $L$ and $L^{\prime}$ and if there is a simplicial isomorphism $h: L \rightarrow L^{\prime}$ such that for every $a \in L$, the linear map determined by $h: a \rightarrow h(a)$ is an isometry (that is, it extends to an isometry of the affine spaces generated by these simplexes). This note is concerned with certain characteristics of a metric complex $M$ which are intrinsic, i.e., which depend only on the isometry class of $M$. The basic such characteristic is the intrinsic metric, which is best described in the piecewise linear context by $\mathrm{H}$. Gluck [3]; for a more general treatment see W. Rinow [8].

Let $M \subseteq R^{l}$ be a metric complex and let $p$ be a point of $M$. Then the tangent cone $T_{p} M$ of $M$ at $p$ is defined to be the infinite cone with vertex $p$ generated by $\operatorname{link}(p, M)$. The isometry class of $T_{p} M$ is intrinsic to $M$, for each $p$. An infinite ray $p \bar{x}$ in $T_{p} M$ will be called a tangent direction at $p$ to $M$.

Let $N_{p} M$ be a subcone of $T_{p} M$ and let $j$ be a nonnegative integer. Let $R^{j} \times N_{p} M$ be given the metric in which its factors are orthogonal. For various choices of $N_{p} M$ and $j, R^{j} \times N_{p} M$ will be isometric to $T_{p} M$. For example if $p$ is in the interior of a $j$-simplex of $M$, such a factoring exists. Consider those factorings of $T_{p} M$ for which $j$ is maximal; then the corresponding $N_{p} M$ are all isometric. Such an $N_{p} M$ will be called the normal geometry of $p$ in $M$, and denoted $v_{p} M$. For example, if $M$ is an $n$-manifold and $p$ is in the interior of an $(n-1)$ - or $n$-simplex, then $v_{p} M=\{p\}$. If $M$ is a 2-manifold, then $v_{p} M=\{p\}$ unless $p$ is a vertex of nonzero curvature, when $v_{p} M=T_{p} M$.

Clearly $j$ and $v_{p} M$ determine the metric geometry of $M$ near $p$.

For any $p \in M$ and any tangent direction $p \bar{x}$ at $p$ lying in $v_{p} M$ I have defined numbers $k_{+}(p \bar{x})$ and $k_{-}(p \bar{x})$, with $k_{+}(p \bar{x}) \geqq k_{-}(p \bar{x})$, called the maximum and minimum curvatures of $M$ at $p$ in the direction $p \bar{x}$. The definitions are too long to give here. Roughly speaking, $k_{-}(p \bar{x})$ equals: $2 \pi$ minus twice the maximum "angle" that can occur between $p \bar{x}$ and any other $p \bar{y} \subseteq v_{p} M$ as $y$ varies; $k_{+}(p \bar{x})$ is defined similarly, using a

AMS (MOS) subject classifications (1970). Primary 53C70, 57C99; Secondary 53C45.

${ }^{1}$ Research supported in part by NSF Grants P22927 and P029431000. 
mini-max. If $M$ is a 2-manifold, then $k_{+}(p \bar{x})=k_{-}(p \bar{x})$ and depends only on $p$; they are both equal to the standard piecewise linear curvature of $M$ at $p$ (see Aleksandroff and Zalgaller [1] or W. Rinow [8]). There seems to be some connection between $k_{-}(p \bar{x})$ and, in the smooth case, the minimum sectional curvature at a point of two-planes containing a given tangent vector at that point; likewise between $k_{+}(p \bar{x})$ and the maximum such sectional curvature. To support this intuition I offer these results:

THEOREM 1. Let $M$ be a complete metric complex such that $k_{+}(p \bar{x}) \leqq 0$ for all $p \in M$ and all tangent directions $p \bar{x} \subseteq v_{p} M$. Then:

(i) for any $p, q \in M$ and any homotopy class $\psi$ of paths from $p$ to $q$ there is exactly one shortest path in $\psi$;

(ii) in particular, if $M$ is simply connected, then it is contractible;

(iii) if $M$ is a simply-connected manifold without boundary of dimension $n \geqq 6$, then $M$ is piecewise linearly isomorphic to Euclidean space $R^{n}$.

Theorem 1 is analogous to a theorem proved for smooth manifolds by E. Cartan [2] under the hypothesis that every sectional curvature be $\leqq 0$.

THEOREM 2. Let $M$ be a complete metric complex which is an n-manifold without boundary. Assume that whenever a is an $(n-2)$-simplex, whenever $p \in$ int $a$ and whenever $p \bar{x} \subseteq v_{p} M$, then $k_{-}(p \bar{x}) \supsetneqq 0$. Then:

(i) if $n$ is even and $M$ orientable, then $M$ is simply connected;

(ii) if $n$ is odd, then $M$ is orientable.

In the smooth case a theorem analogous to (i) was proved by J. Synge [10], and (ii) is an elementary consequence of his method observed by A. Preissman [7].

THEOREM 3. Let $M$ be a complete metric complex which is an $n$-manifold without boundary. Assume:

1. there is a number $k \supsetneqq 0$ such that whenever $a$ is an $(n-2)$-simplex, whenever $p \in$ int $a$ and whenever $p \bar{x} \subseteq v_{p} M$, then $\operatorname{dim} v_{p} M=2$ and $k_{-}(p \bar{x}) \geqq k$;

2. there is a number $Q$ such that whenever $a$ is an $n$-simplex of $M$ and $M$ is represented as a linear complex in $R^{l}$, then the $n$-sphere in $R^{l}$ that passes through the vertices of a has radius $\leqq Q$. Then:

(i) $M$ is compact (I can in fact give a crude estimate for the diameter of $M$ );

(ii) $M$ has positive curvature "everywhere": $k_{-}(p \bar{x}) \supsetneqq 0$ provided that $p$ is not in the interior of an $(n-1)$ - or n-simplex.

Theorem 3 is a weak analogue of a theorem proved for smooth manifolds by S. Myers [6] under the hypothesis that the mean curvature be everywhere bounded above 0. I suspect that the curvature hypothesis of 
Theorem 3 can be weakened once one has the right piecewise linear notion of mean curvature.

An amusing consequence of Theorem 3 is:

THEOREM 4. Let $K$ be a simplicial 3-manifold without boundary. Assume that every 1-simplex is a face of at most five 3-simplexes. Then $K$ is finite.

The proof is to give $K$ a metric by making all the tetrahedra regular of side length 1; then the hypotheses of Theorem 3 are satisfied. A. Phillips has pointed out to me that $R^{3}$ can be triangulated so that every 1 -simplex is a face of at most six 3-simplexes.

Discussion of THEOREM 1. The proof of this theorem is analogous to the proof of Cartan's theorem in the smooth case (see J. Milnor's [5]) The curvature hypothesis on $M$ is equivalent to the hypothesis that $M$ has unique geodesics locally. This means: every $p \in M$ has a neighbourhood $U$ such that whenever $x, y \in U$, then there is a unique geodesic in $M$ from $x$ to $y$. Hence for any $p, q \in M$ one can approximate (as in [5]) the space $\Omega$ of paths from $p$ to $q$ and the energy function $E: \Omega \rightarrow R^{1}$ by a finitedimensional space $V$ and a function $F: V \rightarrow R^{1}$. $F$ is not smooth; nonetheless one can show that $F$ has no "critical points" except local minima. Conclusion (i) follows, as in [5].

In the smooth case one proves (iii) by inferring that at any point $p \in M$ the exponential map $\exp _{p}: T_{p} M \rightarrow M$ is globally defined and is a diffeomorphism. In the piecewise linear case this argument fails, even for 2 -manifolds. However one can consider the function distance-from- $p$ $\rho_{p}: M \rightarrow R^{1}$ and verify that its only "critical point" is $p$. It follows from a theorem of J. Stallings [9] (in the piecewise smooth context) that $M$ is piecewise diffeomorphic to $R^{n}$, and hence from triangulation theory (see M. Hirsch and B. Mazur [4]) that $M$ is piecewise linearly isomorphic to $R^{n}$. At one point in this argument the $h$-cobordism theorem is used to show that certain points are not "critical"; hence the restriction $n \geqq 6$.

Discussion OF THEOREM 3. (The proof of Theorem 2 is quite similar to that of Theorem 3.) The first (curvature) hypothesis on $M$ implies that the whole $(n-2)$-skeleton $M^{n-2}$ is intrinsic to $M$, for it is the coarsest possible triangulation of the "singular set" of $M$-that is, of the set of points where the normal geometry is nontrivial. The second hypothesis then says that the singular set is "fairly dense" in $M$; it implies for example that every point of $M$ is distant at most $Q$ from the singular set.

Let $P$ be a number $\geqq Q$. Let $a$ be a linear simplex in $R^{l}$ which satisfies hypothesis 2 . Let $S$ be an $(l-1)$-sphere with centre $C$ and radius $P$ which passes through the vertices of $a$. Then $C$ does not lie in the affine plane spanned by $a$, so I can project $a$ into $S$ from $C$. Call the image $a \#$; then $a \#$ is the $P$-spherical simplex associated to $a$. Let $\mathscr{M}$ be the simplicial 
complex $M$ re-metrized by replacing each $a \in M$ by the associated $P$-spherical simplex.

The proof of Theorem 3 now falls into four parts. First, whenever $P$ is large enough, then $\mathscr{M}$ satisfies hypothesis 1 (with a different bound $k \# \gtreqless 0$ for the curvature). Second, one shows by induction on $\operatorname{dim} v_{p} M$ that conclusion (ii) holds for $M$ and for $\mathscr{M}$. The inductive step is based on the third part, assumed proved in dimensions $\npreceq n$. The third part is to show that then $\mathscr{M}$ has diameter $\leqq \pi P$. Finally, one has to compare the intrinsic metrics on $M$ and $\mathscr{M}$.

The nub of the proof is the third part. It is proved by inferring from hypothesis 1 for $\mathscr{M}$ that any geodesic $\alpha$ in $\mathscr{M}$ meets the singular set $\mathscr{M}^{n-2}$ at most in the endpoints of $\alpha$. Hence a neighbourhood of $\alpha$ can be immersed isometrically in the standard $n$-sphere $S$ of radius $P$. If $\alpha$ has length $\supsetneqq \pi P$, then its image $\alpha^{\prime}$ in $S$, having the same length as $\alpha$, can be approximated by shorter paths $\beta^{\prime}$ with the same endpoints. But any $\beta^{\prime}$ close enough to $\alpha^{\prime}$ corresponds to a path $\beta$ in $\mathscr{M}$ with the same endpoints as $\alpha$ and the same length as $\beta^{\prime}$. Thus $\alpha$ is not a shortest path; this proves the assertion.

\section{REFERENCES}

1. A. D. Aleksandrov and V. A. Zalgaller, Two-dimensional manifolds of bounded curvature, Trudy Mat. Inst. Steklov. 63 (1962); English transl., Intrinsic geometry of surfaces, Trans. Math. Monographs, vol. 15, Amer. Math. Soc., Providence, R.I., 1967. MR 27 1911.

2. E. Cartan, Leçons sur la géométrie des espaces de Riemann, Gauthier-Villars, Paris, 1928; 2nd ed., 1951. MR 13, 491.

3. H. R. Gluck, Piecewise linear methods in Riemannian geometry, Mimeographed Notes, University of Pennsylvania, Philadelphia, Pa., 1972.

4. M. Hirsch and B. Mazur, Smoothings of piecewise-linear manifolds, Mimeographed Notes, Cambridge, 1964.

5. J. Milnor, Morse theory, Ann. of Math. Studies, no. 51, Princeton Univ. Press, Princeton, N.J., 1963. MR 29 \#634.

6. S. B. Myers, Riemannian manifolds with positive mean curvature, Duke Math. J. 8 (1941), 401-404. MR 3, 18.

7. A. Preissman, Quelques propriétés globales des espaces de Riemann, Comment. Math. Helv. 15 (1943), 175-216. MR 6, 20.

8. W. Rinow, Die innere Geometrie der metrischen Raeume, Die Grundlehren der math. Wissenschaften, Band 105, Springer-Verlag, Berlin, 1961. MR 23 \# A1290.

9. J. R. Stallings, The piecewise-linear structure of Euclidean space, Proc. Cambridge Philos. Soc. 58 (1962), 481-488. MR 26 \#6945.

10. J. L. Synge, On the connectivity of spaces of positive curvature, Quart. J. Math. 7 (1936), 316-320.

Department of Mathematics, State University of New York at Stony Brook, STONY BROOK, NEW YORK 11790 